\title{
Asymptotic Differentiable Structure on Cantor Set
}

\author{
Yunping Jiang \\ Department of Mathematics, Queens College of CUNY, Flushing, NY 11367, USA
}

Received January 17, 1992; in revised form November 3, 1992

\begin{abstract}
We study hyperbolic maps depending on a parameter $\varepsilon$. Each of them has an invariant Cantor set. As $\varepsilon$ tends to zero, the map approaches the boundary of hyperbolicity. We analyze the asymptotics of scaling function of the invariant Cantor set as $\varepsilon$ goes to zero. We show that there is a limiting scaling function of the limiting map and this scaling function has dense jump discontinuities because the limiting map is not expanding. Removing these discontinuities by continuous extension, we show that we obtain the scaling function of the limiting map with respect to a Ulam-von Neumann type metric.
\end{abstract}

\section{Introduction}

A differentiable structure on the topological Cantor set determined by the intrinsic scaling function of a geometric Cantor set on the line has been introduced by Sullivan [4]. Consider a family of geometric Cantor sets on the line. What will happen to such differentiable structures if all gaps of geometric Cantor sets finally close to form an interval (a one dimensional differentiable manifold)? It is the question we will discuss in this paper. The main results (Theorems 1 and 2) say that for a family of geometric Cantor sets on the line generated by a family of nonlinear folding maps, the scaling functions tend to the scaling function of a sequence of partitions on the interval [2] as all gaps finally close. And moreover, the scaling function of the sequence of partitions on the interval has countably many jump discontinuities, and is uniformly continuous at the rest of the points on the dual Cantor set. If we replace all the discontinuities by the continuous extension of the values on the rest of the points, we get a Hölder continuous function on the dual Cantor set. This function is the scaling function of a sequence of partitions with respect to a Ulam-von Neumann type metric [6] (we call this metric an orbifold metric (with respect to the Lebesgue metric)). In the other words, if we consider the interval with this Ulam-von Neumann type metric as a Riemannian manifold (an orbifold, compare to [5]), the limit of differentiable structures on the topological Cantor set is the differentiable structure of this Riemannian manifold. 
This paper is organized as follows: in Sect. 1, we recall a short definition of a scaling function for both the geometric Cantor set on the line and a sequence of partitions on the line. Following the definitions we state the main results (Theorem 1 and 2). In Sect. 2, we show two uniform estimates of the nonlinearities of long compositions of any map in a family (Lemma 2 and 3) and the proofs of the main results.

\section{Statements of Main Results}

Suppose $I$ is the interval $[-1,1]$ and $f$ is a $C^{1}$ folding map from $I$ to the real line with a unique critical point 0 . We say 0 is a power law critical point if there is a number $\gamma>1$ such that the limits of $f^{\prime}(x) /|x|^{\gamma-1}$ exist and equal nonzero numbers as $x$ tends to zero from above and below. The number $\gamma$ is called an exponent of $f$. Henceforth, we will assume that $f$ is increasing on $[-1,0]$ and decreasing on $[0,1]$, and $f(0) \geq 1, f(-1)=f(1)=-1$, and that 0 is the power law critical point of $f$.

Let $g_{0}$ from $I$ to $[-1,0]$ and $g_{1}$ from $I$ to $[0,1]$ be the left and right inverse branches of $f$. For a sequence $w=i_{0} \ldots i_{n}$ of symbols $\{0,1\}$, let $g_{w}=g_{i_{0}} \circ \ldots \circ g_{i_{n}}$ be the composition of $g_{i_{0}}$ to $g_{i_{n}}$ and $I_{2}=g_{w}(I)$ be the image of $I$ under $g_{w}$. We use $\eta_{n}$ to denote the collection of intervals $I_{w}$ for all $w$ of length $n+1$ and $\lambda_{n}=\max _{I_{w} \in \eta_{n}}\left|I_{w}\right|$ to denote the maximum of the lengths of the intervals in $\eta_{n}$.

Definition 1. We say $f$ is in $\mathscr{H}$, the space of hyperbolic maps, if (a) $f(0)>1$, (b) $f$ is $C^{1+\alpha}$ for some $0<\alpha \leq 1$, and (c) there are constants $K>0$ and $0<\mu<1$ such that $\lambda_{n} \leq K \mu^{n}$ for all integers $n>0$.

Suppose $f$ is a map in $\mathscr{H}$. Then $C_{f}=\bigcap_{n=0}^{+\infty} f^{-n}(I)$, the maximal invariant set of $f$, is a geometric Cantor set on the line (the proof is to use the naive distortion lemma). The scaling function of this geometric Cantor set has been defined in [4] as follows: suppose $w_{n}=i_{n} \ldots i_{1} i_{0}$ is a finite sequence of 0 and 1 . Take $v_{n-1}=i_{n} \ldots i_{1}$ and define $s\left(w_{n}\right)=\left|I_{w_{n}}\right| /\left|I_{v_{n-1}}\right|$, where $I_{w_{n}}=g_{w_{n}}(I), I_{v_{n-1}}=g_{v_{n-1}}(I)$ and $|\cdot|$ is the Lebesgue measure. Suppose

$$
C^{*}=\left\{a^{*}=\ldots i_{n} \ldots i_{1} i_{0} \mid i_{n} \in\{0,1\}\right\}
$$

is the dual Cantor set. If $\lim _{n \mapsto+\infty} s\left(w_{n}\right)$ exists for every $a^{*}=\ldots w_{n}$ in $C^{*}$, we define a function $s\left(a^{*}\right)=\lim _{n \mapsto+\infty} s\left(w_{n}\right)$ on $C^{*}$. This function is the scaling function of $C_{f}$. It is a $C^{1}$-invariant (in other words, it is the same for both $f$ and $h \circ f \circ h^{-1}$ whenever $h$ is a $C^{1}$-diffeomorphism of $I$ ).

Theorem A (Sullivan [4]). Suppose $f$ is a map in $\mathscr{H}$. The scaling function $s$ of $C_{f}$ exists and is Hölder (see [1] for the definition of a Hölder continuous function on a symbolic space).

Now we consider a map $f$ with $f(0)=1$, which maps $I$ into itself. In this case, the interval $I$ is the maximal invariant set of $f$ and we define an orbifold metric on $I$ (associated with $f$ ) as

$$
d y=\frac{d x}{\left(1-x^{2}\right)^{\frac{\gamma-1}{\gamma}}} .
$$


Remark 1. The orbifold metric $d y$ can be thought of as a singular metric with respect to the Lebesgue metric $d x$ and the corresponding change of coordinate is $h_{\eta}(x)=-1+b \int_{-1}^{t} d t /\left(1-t^{2}\right)^{(\gamma-1) / \gamma}$, where $b$ is determined by $h(1)=1$.

Lemma 1. Suppose $\tilde{f}=h_{\gamma} \circ f \circ h_{\gamma}^{-1}$ (it is $f:(I, d y) \mapsto(I, d y)$ ). Then $\tilde{f}$ is continuous and $\tilde{f} \mid[-1,0]$ and $\tilde{f} \mid[0,1]$ are $C^{1}$-embeddings.

Proof. It is clearly that $\tilde{f}$ is continuous and $\tilde{f}^{\prime}(x) \neq 0$ for $x$ in $(0,1)$. By the definition of the power law critical point in this paper, it is easy to check that the one-sided derivatives of $\tilde{f}(x)$ at $0,-1$ and 1 are not zero either, and that $\tilde{f}^{\prime}(x)$ is continuous on $[-1,0]$ and $[0,1]$.

Definition 2. We say $f$ is on $\mathscr{B} H$, the boundary of hyperbolicity, if (1) $f(0)=1$, (2) $\tilde{f} \mid[-1,0]$ and $\tilde{f} \mid[0,1]$ are $C^{1+\alpha}$ embeddings for some $0<\alpha \leq 1$, and (3) there are constants $K>0$ and $0<\mu<1$ such that $\lambda_{n} \leq K \mu^{n}$ for all integers $n>0$.

Remark 2. Let $r_{-}(x)$ and $r_{+}(x)$ be $f^{\prime}(x) /|x|^{\gamma-1}$ on $[-1,0]$ and $[0,1]$, respectively. Then (2) is equivalent to say that $f, r_{-}$and $r_{+}$are $C^{1+\alpha^{\prime}}$ for some $0<\alpha^{\prime} \leq 1$. Some examples of maps on $\mathscr{B} H$ can be found in [3].

Although the maximal invariant set of $f$ on $\mathscr{B} H$ is the interval $I$, we can still define the scaling function of the sequence of partitions of $I$ similarly: suppose $\eta=\left\{\eta_{n}\right\}_{n=0}^{+\infty}$ is the sequence of partitions of $I$ determined by $f$. For every finite sequence $w_{n}=i_{n} \ldots i_{1} i_{0}$ of 0 and 1 , take $v_{n-1}=i_{n} \ldots i_{1}$ and define $s\left(w_{n}\right)=\left|I_{w_{n}}\right| /\left|I_{v_{n-1}}\right|$, where $I_{w_{n}}=g_{w_{n}}(I), I_{v_{n-1}}=g_{v_{n-1}}(I)$ and $|\cdot|$ is the Lebesgue measure. If $\lim _{n \mapsto+\infty} s\left(w_{n}\right)$ exists for every $a^{*}=\ldots w_{n}$ in $C^{*}$, we define a function $s\left(a^{*}\right)=\lim _{n \mapsto+\infty} s\left(w_{n}\right)$ on $C^{*}$. This function is the scaling function of $\eta$. It is again a $C^{1}$-invariant.

Let $I^{*}=\left\{a^{*}=\ldots i_{n} \ldots i_{0} \mid \in C^{*}, i_{n_{k}}=1\right.$ for infinitely many $\left.k\right\}$ and $O^{*}$ be the complement of $I^{*}$ in $C^{*}$. The set $O^{*}$ is countable.

Theorem 1. Suppose $f$ is a map on . R $H$ and $\eta=\left\{\eta_{n}\right\}_{n=0}^{+\infty}$ is the sequence of partitions of $I$ determined by $f$. The scaling function $s$ of $\eta$ exists, and is continuous at every point $a^{*}$ in $I^{*}$ and discontinuous at every point $a^{*}$ in $O^{*}$. Moreover, every $a^{*}$ in $O^{*}$ is a jump discontinuity of $s$ and the restriction $s \mid I^{*}$ is uniformly continuous (see Fig. 1).
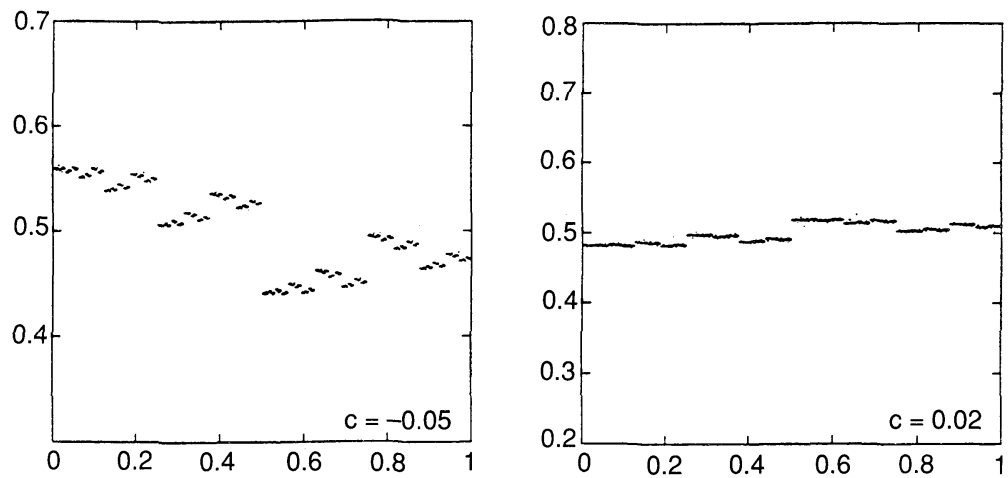

Fig. 1. The graphs of the scaling functions for $f_{c}(x)=-x^{2}+2+c x^{2}\left(4-x^{2}\right)$ 
Suppose $f$ is on $\mathscr{B} H$ and $\eta=\left\{\eta_{n}\right\}_{n=0}^{+\infty}$ is the sequence of partitions of $I$ determined by $f$. Let $\tilde{s}\left(w_{n}\right)=\left\|I_{w_{n}}\right\| /\left\|I_{v_{n-1}}\right\|$, where $w_{n}=v_{n-1} i_{0}$ and $\|\cdot\|$ is the measure on $I$ induced by the orbifold metric $d y$. If $\tilde{s}\left(a^{*}\right)=\lim _{n \mapsto+\infty} \tilde{s}\left(w_{n}\right)$ exists for every $a^{*}=\ldots w_{n}$ in $C^{*}$, then $\tilde{s}$ is defined as the scaling function of $\eta$ under $d y$.

Corollary 1. Suppose $f$ is on $\mathscr{B} H$. The scaling function $\tilde{s}$ of $\eta$ under the orbifold metric dy exists and is Hölder, and moreover, it is the continuous extension of $s \mid I^{*}$ to $C^{*}$ (see Fig. 1).

A family $\left\{f_{\varepsilon}\right\}_{0 \leq \varepsilon \leq 1}$ in $\mathscr{H} \cup \mathscr{B} H$ with $f_{\varepsilon}(0)=1+\varepsilon$ is said to be regular if

(i) $F(x, \varepsilon)=f_{\varepsilon}(x)$ is a $C^{1}$ function of $[-1,1] \times[0,1]$ and $f_{\varepsilon}^{\prime}(x)$ are uniformly $\alpha$-Hölder continuous functions of $x$,

(ii) $f_{\varepsilon}$ has the same exponent $\gamma$ at 0 for every $\varepsilon$ in $[0,1]$ and $r_{-}(x, \varepsilon)=f_{\varepsilon}^{\prime}(x) /|x|^{\gamma-1}$ on $[-1,0] \times[0,1]$ and $r_{+}(x, \varepsilon)=f_{\varepsilon}^{\prime}(x) /|x|^{\gamma-1}$ on $[0,1] \times[0,1]$ are continuous and $r_{+}(x, \varepsilon)$ and $r_{-}(x, \varepsilon)$ are uniformly $\alpha$-Hölder continuous functions of $x$, and

(iii) there are two constants $K>0$ and $0<\mu<1$ such that $\lambda_{n, \varepsilon} \leq K \mu^{n}$ for all $n>0$ and $0 \leq \varepsilon \leq 1$.

Theorem 2. Suppose $\left\{f_{\varepsilon}\right\}_{0 \leq \varepsilon \leq 1}$ in $\mathscr{H} \cup \mathscr{B} H$ is regular and $\left\{s_{\varepsilon}\right\}_{0 \leq \varepsilon \leq 1}$ is the family of corresponding scaling functions. Then

(I) for $\varepsilon_{0} \in(0,1], s_{\varepsilon}$ converges to $s_{\varepsilon_{0}}$ uniformly on $C^{*}$ as $\varepsilon$ tends to $\varepsilon_{0}$,

(II) for every $a^{*} \in C^{*}, s_{\varepsilon}\left(a^{*}\right)$ tends to $s_{0}\left(a^{*}\right)$ as $\varepsilon$ goes to zero, and

(III) $s_{\varepsilon} \mid I^{*}$ converges to $s_{0} \mid I^{*}$ as $\varepsilon$ goes to zero.

\section{The Proofs of the Main Results}

Suppose $\left\{f_{\varepsilon}\right\}_{0 \leq \varepsilon \leq 1}$ in $\mathscr{H} \cup \mathscr{B} H$ with $f_{\varepsilon}(0)=1+\varepsilon$ is a regular family. Let $U_{\varepsilon}$ be the interval bounded by $f_{\varepsilon}^{-1}(0)$ and $V_{\varepsilon}$ be the closure of the complement of $U_{\varepsilon}$ in $I$ for every $\varepsilon$ in $[0,1]$ (see Fig. 2).

Fig. 2

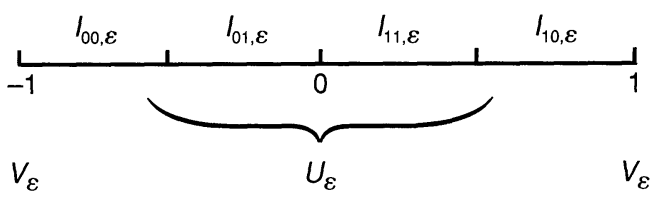

Lemma 2. There is a constant $K>0$ such that for every $\varepsilon$ in $[0,1]$, if $f_{\varepsilon}^{\circ i}(x)$ and $f_{\varepsilon}^{\circ i}(y)$ are in the same connected component of $V_{\varepsilon}$ for $i=0,1, \ldots, n-1$, then

$$
\frac{\left|\left(f_{\varepsilon}^{\circ n}\right)^{\prime}(x)\right|}{\left|\left(f_{\varepsilon}^{\circ n}\right)^{\prime}(y)\right|} \leq \exp \left(K\left|f_{\varepsilon}^{\circ n}(x)-f_{\varepsilon}^{\circ n}(y)\right|^{\alpha}\right) \text {. }
$$

Proof. It is the naive distortion lemma since $f_{\varepsilon}^{\prime}(x) \neq 0$ for $x$ in $V_{\varepsilon}$.

Lemma 3. There is a constant $K>0$ such that for every $\varepsilon$ in $[0,1]$, every pair $x$ and $y$ in an interval of $\eta_{m, \varepsilon}$ and every $0<n \leq m$, if $f_{\varepsilon}^{\circ n}(x)$ and $f_{\varepsilon}^{\circ n}(y)$ are in $U_{\varepsilon}$, then

$$
\frac{\left|\left(f_{\varepsilon}^{\circ n}\right)^{\prime}(x)\right|}{\left|\left(f_{\varepsilon}^{\circ n}\right)^{\prime}(y)\right|} \leq \exp \left(K\left|f_{\varepsilon}^{\circ n}(x)-f_{\varepsilon}^{\circ n}(y)\right|^{\alpha}\right) \text {. }
$$


Proof. Let $x_{\imath}$ and $y_{\imath}$ be the images of $x$ and $y$ under $f_{\varepsilon}^{\circ i}$. Then $x_{i}$ and $y_{i}$ lie in a same interval of $\eta_{(m-i), \varepsilon}$. For every positive integer $n \leq m$,

$$
\frac{\left(f_{\varepsilon}^{\circ n}\right)^{\prime}(x)}{\left(f_{\varepsilon}^{\circ n}\right)^{\prime}(y)}=\prod_{i=0}^{n-1} \frac{f_{\varepsilon}^{\prime}\left(y_{i}\right)}{f_{\varepsilon}^{\prime}\left(x_{i}\right)} .
$$

This product can be factored into two products,

$$
\prod_{x_{i}, y_{\imath} \in V_{\varepsilon}} \frac{f_{\varepsilon}^{\prime}\left(y_{i}\right)}{f_{\varepsilon}^{\prime}\left(x_{i}\right)} \text { and } \prod_{x_{\imath}, y_{\imath} \in V_{\varepsilon}} \frac{f_{\varepsilon}^{\prime}\left(y_{i}\right)}{f_{\varepsilon}^{\prime}\left(x_{\imath}\right)} .
$$

Since (i) and (iii) in the definition of a regular family, there is a constant $K_{1}>0$ such that

$$
\prod_{x_{\imath}, y_{\imath} \in V_{\varepsilon}} \frac{\left|f_{\varepsilon}^{\prime}\left(y_{i}\right)\right|}{\left|f_{\varepsilon}^{\prime}\left(x_{i}\right)\right|} \leq K_{1}
$$

To estimate the second product, let

$$
h_{\varepsilon}(x)=-1+b_{\varepsilon} \int_{-1}^{x} \frac{d t}{\left((1+\varepsilon)-t^{2}\right)^{\frac{\gamma-1}{\gamma}}}
$$

be the family of changes of coordinates on $I$ (where $h_{0}$ is the orbifold metric we mentioned in Sect. 1). Let

$$
\tilde{f}_{\varepsilon}(x)=h_{\varepsilon}^{-1} \circ f_{\varepsilon} \circ h_{\varepsilon}(x) .
$$

Then we can factor the second product into three factors,

$$
\prod_{x_{i}, y_{\imath} \in U_{\varepsilon}} \frac{f_{\varepsilon}^{\prime}\left(y_{\imath}\right)}{f_{\varepsilon}^{\prime}\left(x_{i}\right)}=\prod_{x_{i}, y_{\imath} \in U_{\varepsilon}} \frac{h_{\varepsilon}^{\prime}\left(y_{i}\right)}{h_{\varepsilon}^{\prime}\left(x_{i}\right)} \cdot \prod_{x_{i}, y_{i} \in U_{\varepsilon}} \frac{\tilde{f}_{\varepsilon}^{\prime}\left(h_{\varepsilon}\left(y_{i}\right)\right)}{\tilde{f}_{\varepsilon}^{\prime}\left(h_{\varepsilon}\left(x_{\imath}\right)\right)} \cdot \prod_{x_{\imath}, y_{\imath} \in U_{\varepsilon}} \frac{h_{\varepsilon}^{\prime}\left(f\left(x_{i}\right)\right)}{h_{\varepsilon}^{\prime}\left(f\left(y_{\imath}\right)\right)} .
$$

Further, the last one can be factored into

$$
\prod_{x_{\imath}, y_{i} \in U_{\varepsilon}} \frac{\left(1+f_{\varepsilon}\left(y_{\imath}\right)\right)^{\frac{\gamma-1}{\gamma}}}{\left(1+f_{\varepsilon}\left(x_{i}\right)\right)^{\frac{\gamma-1}{\gamma}}} \text { and } \prod_{x_{i}, y_{i} \in U_{\varepsilon}} \frac{\left(1-f_{\varepsilon}\left(y_{i}\right)\right)^{\frac{\gamma-1}{\gamma}}}{\left(1-f_{\varepsilon}\left(x_{i}\right)\right)^{\frac{\gamma-1}{\gamma}}} .
$$

Since $h_{\varepsilon}^{\prime}(x)$ and $\tilde{f}_{\varepsilon}^{\prime}(x)$ are uniformly $\alpha^{\prime}$-Hölder continuous functions on each of $I_{01, \varepsilon}$ and $I_{11, \varepsilon}\left(U_{\varepsilon}=I_{01, \varepsilon} \cup I_{11, \varepsilon}\right)$, there is a constant $K_{2}>0$ so that

$$
\prod_{x_{i}, y_{\imath} \in U_{\varepsilon}} \frac{\left|h_{\varepsilon}^{\prime}\left(y_{2}\right)\right|}{\left|h_{\varepsilon}^{\prime}\left(x_{i}\right)\right|} \leq K_{2} \quad \text { and } \quad \prod_{x_{i}, y_{i} \in U_{\varepsilon}} \frac{\left|\tilde{f}_{\varepsilon}^{\prime}\left(h_{\varepsilon}\left(y_{i}\right)\right)\right|}{\left|\tilde{f}_{\varepsilon}^{\prime}\left(h_{\varepsilon}\left(x_{i}\right)\right)\right|} \leq K_{2} .
$$

It is clearly that there is a constant $K_{2}>0$ such that

$$
\prod_{x_{\imath}, y_{\imath} \in U_{\varepsilon}} \frac{\left(1+f_{\varepsilon}\left(y_{i}\right)\right)^{\frac{\gamma-1}{\gamma}}}{\left(1+f_{\varepsilon}\left(x_{i}\right)\right)^{\frac{\gamma-1}{\gamma}}} \leq K_{3} .
$$

To finish the proof we write

$$
\frac{1-f_{\varepsilon}\left(y_{i}\right)}{1-f_{\varepsilon}\left(x_{i}\right)}=1+\frac{f_{\varepsilon}\left(x_{i}\right)-f_{\varepsilon}\left(y_{\imath}\right)}{1-f_{\varepsilon}\left(x_{i}\right)} .
$$


From the facts that $f_{\varepsilon}(1)=f_{\varepsilon}(-1)=-1$ and $f^{\circ j}\left(x_{i}\right)$ is in $U_{\varepsilon}$ for some $i<j \leq n$ (which is assured by the assumption of the lemma), we can find a constant $K_{4}>0$ such that

$$
\prod_{x_{i}, y_{i} \in U_{\varepsilon}} \frac{\left(1-f_{\varepsilon}\left(y_{i}\right)\right)^{\frac{\gamma-1}{\gamma}}}{\left(1-f_{\varepsilon}\left(x_{i}\right)\right)^{\frac{\gamma-1}{\gamma}}} \leq K_{4} .
$$

Then $K=K_{1} K_{2}^{2} K_{3} K_{4}$ is a constant which satisfies the lemma.

A straightforward calculation from Lemma 2 and 3 to the cases that $a^{*}=\ldots w_{n}$ is in $O^{*}$ and is in $I^{*}$, respectively, implies that there are constants $K=K\left(a^{*}\right)>0$ and $0<\alpha \leq 1$ and a subsequence $\left\{n_{i}\right\}$ of the integers such that for all $m \geq k \geq n_{i}$, and $\varepsilon$ and $\varepsilon^{\prime}$ in $[0,1]$,

$$
\left|s_{\varepsilon}\left(w_{k}\right)-s_{\varepsilon^{\prime}}\left(w_{m}\right)\right| \leq K\left(\left|I_{w_{n_{\imath}}, \varepsilon}\right|^{\alpha}+\left|I_{w_{n_{i}}, \varepsilon^{\prime}}\right|^{\alpha}\right)+\left|s_{\varepsilon}\left(w_{n_{\imath}}\right)-s_{\varepsilon^{\prime}}\left(w_{n_{\imath}}\right)\right|
$$

Proof of Theorem 1. The existence of the scaling function $s$ of the sequence of partition $\eta$ follows from the inequality $(*)$ as $\varepsilon=\varepsilon^{\prime}=0$.

The scaling function $s$ is continuous at every point $a^{*}$ in $I^{*}$ and the restriction $s \mid I^{*}$ is uniformly continuous follow from the inequality $(*)$ and the fact that sup $K\left(a^{*}\right)<\infty$ too. $a^{*} \in I^{*}$

Suppose $a^{*}=(\ldots 00 w i)$ is in $O^{*}$. Let $0_{n}$ be the finite string of zeroes of length $n$. Then the interval $I_{0_{n} w}$ is eventually in the left interval $I_{00}$. We use $b_{n}$ and $a_{n}$ to denote the lengths of $I_{0_{n} w}$ and $I_{0_{n} w i}$, respectively (where $I_{0_{n} w i} \subset I_{0_{n} w}$ ). Let $c_{n}$ be the distance from $I_{0_{n} w}$ to -1 (see Fig. 3).

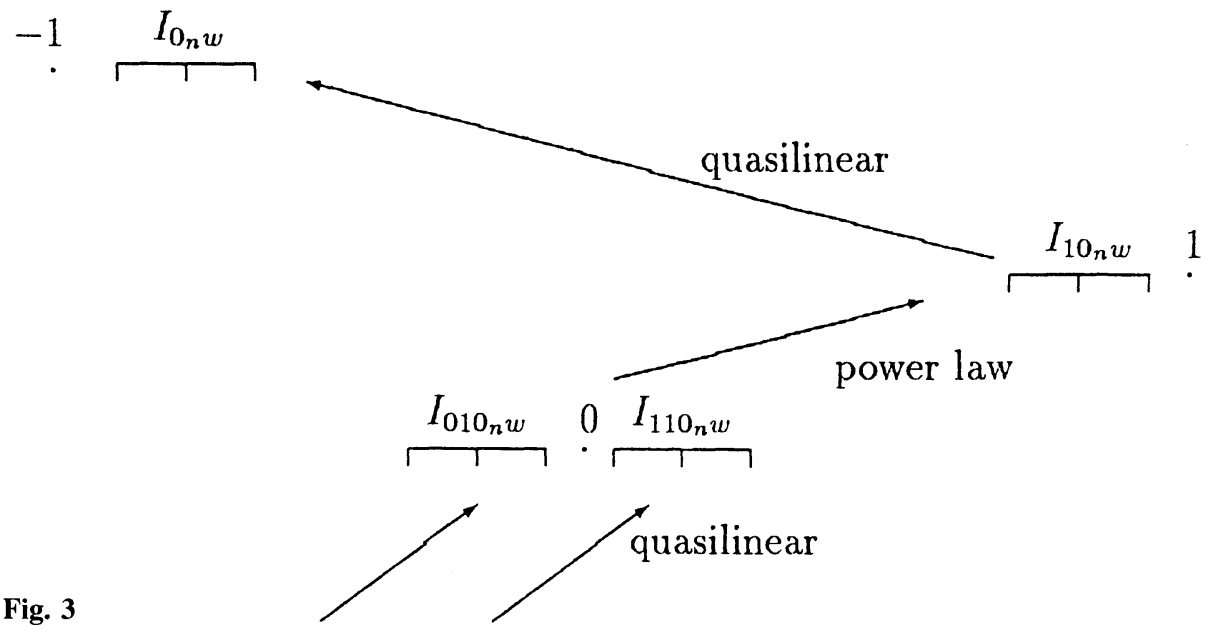

Using the naive distortion lemma, we can show

$$
\tau_{1}\left(a^{*}\right)=\lim _{n \mapsto \infty} \frac{b_{n}}{c_{n}}>\tau_{2}\left(a^{*}\right)=\lim _{n \mapsto \infty} \frac{a_{n}}{c_{n}}
$$


exist. These implies that the limit of the sequence $\left\{s\left(j 10_{n} w i\right)\right\}_{n=1}^{\infty}$ (where $j$ is either 0 or 1) exists and equals

$$
\frac{\left(1+\tau_{2}\left(a^{*}\right)\right)^{\frac{1}{\gamma}}-1}{\left(1+\tau_{1}\left(a^{*}\right)\right)^{\frac{1}{\gamma}}-1} \text { or } \frac{\left(1+\tau_{1}\left(a^{*}\right)\right)^{\frac{1}{\gamma}}-\left(1+\tau_{2}\left(a^{*}\right)\right)^{\frac{1}{\gamma}}}{\left(1+\tau_{1}\left(a^{*}\right)\right)^{\frac{1}{\gamma}}-1}
$$

For a point $b^{*}=\left(\ldots j 10_{n} w i\right)$ in $C^{*}$, because $I_{j 10_{n} w}$ is in $U_{\varepsilon}$ we can use Lemma 3 to show that the error of $s\left(j 10_{n} i\right)$ to $s\left(b^{*}\right)$ can be estimated by $\left|I_{j 10_{n} w i}\right|^{\alpha}$, that is, there is a positive constant $K_{1}$ such that

$$
\left.\mid s\left(b^{*}\right)\right)-\left.s\left(j 10_{n} w i\right)\left|\leq K_{1}\right| I_{j 10_{n} w i}\right|^{\alpha} .
$$

Further,

$$
\lim _{b^{*} \neq a^{*}, b^{*} \mapsto a^{*}} s\left(b^{*}\right)=\lim _{n \mapsto \infty} s\left(j 10_{n} w i\right) .
$$

Since $s\left(a^{*}\right)=\tau_{2}\left(a^{*}\right) / \tau_{1}\left(a^{*}\right)$, we get

$$
\lim _{b^{*} \neq a^{*}, b^{*} \mapsto a^{*}} s\left(b^{*}\right) s\left(b^{*}\right) \neq s\left(a^{*}\right),
$$

in the other words, $s$ has jump discontinuity at $a^{*}$.

Proof of Corollary 1. It follows from the naive distortion lemma and the fact that $\tilde{s}\left|I^{*}=s\right| I^{*}$.

Proof of Theorem 2. (I) is also from the naive distortion lemma. (II) follows from the inequality $(*)$. (III) follows from the inequality $(*)$ and that $0<\max _{a^{*} \in 1^{*}} K\left(a^{*}\right)$ $<+\infty$.

Acknowledgement. I would like to thank Dennis Sullivan for valuable suggestions and remarks.

\section{References}

1. Bowen, R.: Equilibrium states and the ergodic theory of Anosov diffeomorphisms. Berlin, Heidelberg, New York: Springer

2. Jiang, Y.: Ratio geometry in dynamical systems. Preprint, June, 1989, IHES

3. Jiang, Y.: Generalized Ulam-von Neumann transformations. Thesis, Graduate School of CUNY, 1990 and UMI dissertation information service

4. Sullivan, D.: Differentiable Structure on Fractal Like Sets Determined by Intrinsic Scaling Functions on Dual Cantor Sets. The Proceedings of Symposia in Pure Mathematics, Vol. 48, 1988

5. Thurston, W.: The geometry and topology of three-manifolds. Preprint, Princeton University 1982

6. Ulam, S.M., von Neumann, J.: On the Combinations of Stochastic and Deterministic Processes. Bull. Am. Math. Soc. 53, 1120 (1947) 
